\title{
Ionic Bonding
}

National Cancer Institute

\section{Source}

National Cancer Institute. Ionic Bonding. NCI Thesaurus. Code C73475.

Formation of a chemical bond following a transfer of electrons between two ions with opposite charges. 